Classification

Physics Abstracts

$68.25-68.48-81.60 \mathrm{H}$

\title{
Influence d'interphases pyrocarbone déposées par CVI pulsée sur les caractéristiques mécaniques de matériaux composites unidirectionnels
}

\author{
P. Dupel, J.L. Bobet, R. Pailler et J. Lamon \\ Laboratoire des Composites thermostructuraux UMR-47 CNRS-SEP-UB1, Domaine Universi- \\ taire, 3 allée de La Boëtie, 33600 Pessac, France
}

(Reçu le 10 septembre 1993, révisé le 1 mars 1995, accepté le 12 avril 1995)

Résumé. - La CVI pulsée (P-CVI) permet de réaliser de manière controlée des dépôts de Pyrocarbone $(\mathrm{PyC})$ à microstructure anisotrope. Des composites unidirectionnels $\mathrm{SiC} / \mathrm{PyC} / \mathrm{SiC}$ et $\mathrm{C} / \mathrm{PyC} / \mathrm{SiC}$, possédant ce type d'interphase ont été élaborés. Leurs propriétés mécaniques ont été comparées à celles mesurées sur des composites semblables mais possédant une interphase PyC déposée par la voie CVI conventionnelle. Les résultats des essais en traction ont montré : (i) que les caractéristicues ruécaniques pouvaient être notablement augmentées grâce à l'utilisation de la P-CVI, (ii) que l'optimum d'épaisseur de l'interphase PyC (P-CVI) est de l'ordre de 0,2 $\mu \mathrm{m}$ dans le cas de comrecistes unidirectionnels $\mathrm{SiC} / \mathrm{PyC} / \mathrm{SiC}$, (iii) qu'il est en partie possible de corréler les caractéristiciues mécaniques, d'une part, avec les contraintes résiduelles d'origine thermique créées lors de l'élaboration des CMC et dépendant notamment de l'épaisseur de l'interphase, et d'autre part, avec la nature plus orientée du PyC déposé en P-CVI.

\begin{abstract}
Pulse-CVI (P-CVI) allows controlled deposition of anisotropic carbon layers. Unidirectional $\mathrm{SiC} / \mathrm{PyC} / \mathrm{SiC}$ and $\mathrm{C} / \mathrm{PyC} / \mathrm{SiC}$ composites possessing carbon interphases deposited by P-CVI were developed. Tensile mechanical properties were compared to those measured on composite specimens possessing PýC interphases deposited by conventional CVI. Comparison of test data showed that: (i) improved mechanical properties were observed on those specimens with P-CVI interphases, (ii) thickness of $0.2 \mu \mathrm{m}$ for the P-CVI interfacial coating maximizes the material's mechanical properties, (iii) improvement of mechanical properties were correlated with the thermally induced residual stresses that built up during cooling down from the processing temperature and with the oriented microstructures of P-CVI PyC.
\end{abstract}

\section{Introduction}

Les composites à matrice céramique renforcée par des fibres longues sont une classe de matériaux prometteurs [1]. L'introduction d'un renfort fibreux leur confère un caractère endommageable. Différents mécanismes d'endommagement interviennent alors - tels que la multifissuration matricielle et la décohésion fibre-matrice [2] - et permettent d'obtenir des caractéristiques 
mécaniques intéressantes. Cependant, l'existence de ces mécanismes d'endommagement est conditionnée par la qualité de la liaison fibre-matrice. Dans le cas d'une liaison trop forte, aucun des phénomènes cités plus haut n'est observé et le comportement mécanique est purement fragile. Par contre, une liaison trop faible limite les transferts de charge à l'interface et ne permet qu'une faible libération d'énergie par décohésion ce qui se traduit par une contrainte à rupture faible associée à une déformation à rupture élevée. Afin d'optimiser les transferts de charge, et d'obtenir une contrainte et une déformation à rupture élevées, un troisième constituant est souvent déposé entre les fibres et la matrice : l'interphase.

Les matériaux d'interphase les plus fréquemment utilisés sont le pyrocarbone [3] et le nitrure de bore hexagonal [4]. Ces deux matériaux possédent des caractéristiques très voisines : (i) une faible rigidité (50 GPa) comparée à celles des autres constituants du composite et (ii) une structure lamellaire fortement anisotrope. De plus, l'épaisseur de l'interphase exerce une influence importante sur les propriétés mécaniques du composite comme l'ont montré Ménessier et al. [5] dans le cas d'un composite SiC/vitrocéramique ou Lowden \& Stinton [6] et Naslain [7] dans le cas de composites ex-PCS/PyC/SiC testés respectivement en flexion et en traction.

La méthode la plus couramment employée pour déposer une interphase est l'infiltration chimique en phase vapeur (CVI). Cette méthode produit le plus souvent une interphase de qualité plus ou moins controlée dans l'épaisseur [8].

Dupel et al. ont montré que la P-CVI de carbone à partir de propane permet, selon les conditions expérimentales, de contrôler la microstructure et l'épaisseur du PyC déposé et ceci même au sein de pores modèles $[9,10]$.

L'objectif de ce travail est double : d'une part, évaluer les caractéristiques mécaniques de composites unidirectionnels dont l'interphase $\mathrm{PyC}$ a été déposée soit par I-CVI, soit par PCVI, et d'autre part, définir dans le cas d'interphases PyC (P-CVI) l'optimum en épaisseur conduisant aux caractéristiques mécaniques maximales.

\section{Procédure expérimentale}

2.1. Préparation des composites unidirectionnels, - Les matériaux composites unidirectionnels ont été réalisés à partir de deux types de mèches : des mèches de fibres de carbone T300 $\left({ }^{1}\right)$ composées de 1000 filaments, et des mèches de fibres ex-PCS $\left({ }^{2}\right)$ composées de 500 filaments. L'élaboration se décompose en 4 étapes successives :

- (i) le maintien en tension des mèches de $50 \mathrm{~mm}$ de longueur sur des cadres en graphite par une colle céramique,

- (ii) le désensimage des mèches par pyrolyse à $500^{\circ} \mathrm{C}$ sous vide pendant 4 heures,

- (iii) le dépôt de l'interphase pyrocarbone à partir de propane selon des procédures décrites par ailleurs $[9,11]$. Quelle que soit la méthode, le PyC a été déposé sous une pression de $3 \mathrm{kPa}$ et une température de $1000^{\circ} \mathrm{C}$. En I-CVI le débit a été fixé à $60 \mathrm{sccm}$, et en P-CVI la durée du palier à $10 \mathrm{~s}$, le nombre de pulses varie selon l'épaisseur de l'interphase ( $\left.e_{\mathrm{i}}\right)$ déposée (20 pulses pour $e_{\mathrm{i}}=50 \mathrm{~nm}, 800$ pulses pour $e_{\mathrm{i}}=1000 \mathrm{~nm}$ ). Les figures 1 et 2 montrent, à titre d'exemples, des interphases déposées par P-CVI sur des fibres de carbone et de ex-PCS (la matrice, à ce stade, n'est pas encore déposée). L'épaisseur des interphases a été mesurée à l'aide d'un microscope à effet de champ sur des sections droites polies des composites unidirectionnels, l'erreur sur la mesure étant de l'ordre de quelques nanomètres.

$\left({ }^{1}\right)$ fournies par la SEP-Bordeaux

$\left({ }^{2}\right)$ Nicalon NL202 de NIPPON Carbon 


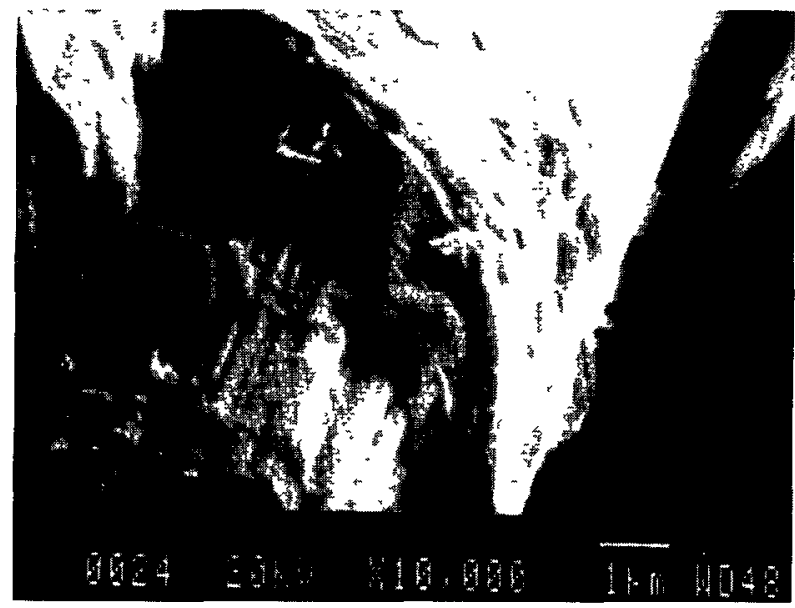

Fig. 1. - Observation au MEB de l'interphase pyrocarbone I-CVI sur une fibre carbone T300 (lot C). [SEM micrograph showing a pyrocarbon interphase deposited by I-CVI on a T300 carbon fiber (batch C).]

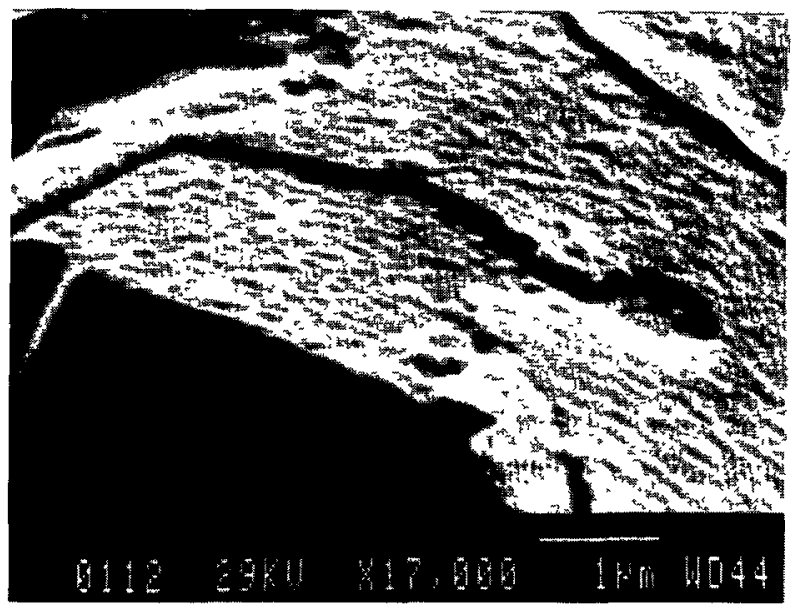

Fig. 2. - Observation au MEB de l'interphase pyrocarbone P-CVI sur une fibre ex-PCS (lot E). [SEM micrograph showing a pyrocarbon interphase deposited by P-CVI on an ex-PCS fiber (batch E).]

- (iv) le dépôt par I-CVI de la matrice SiC sur les mèches dans un four industriel $\left({ }^{3}\right)$ [12]. La figure 3 montre la surface de rupture d'une mèche composite T300/PyC/SiC obtenue dans ces conditions.

Le tableau I rassemble les caractéristiques des différents lots (A à I) de mèches testés lors de cette étude. Les fractions volumiques moyennes de fibres, $\left(v_{\mathrm{f}}\right)$, de matrice, $\left(v_{\text {mat }}\right)$, et d'interphase, $\left(v_{\text {int }}\right)$, ont été calculées à partir de la masse de chaque mèche infiltrée, $\left(m_{\text {mèche }}\right)$, à

$\left({ }^{3}\right)$ selon un procédé développé par la S.E.P 


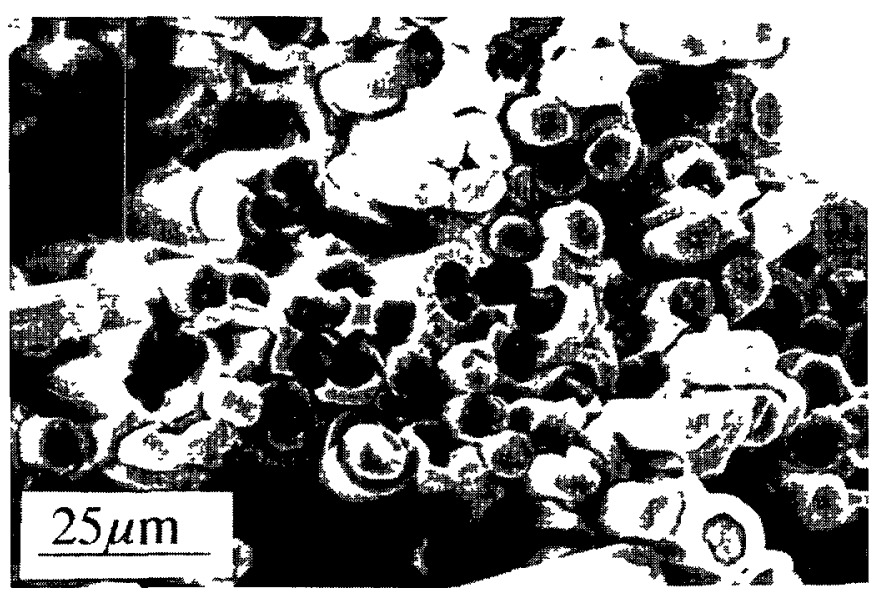

Fig. 3. - Observation au MEB d'une mèche composite (T300/PyC/SiC, lot $\mathrm{C}$ ). [SEM micrograph showing a T300/PyC/SiC bundle (batch $\mathrm{C}$ ).]

Tableau I. - Epaisseur de l'interphase pyrocarbone, mode de dépôt, et pourcentages volumiques moyens de matrice, de fibres, et d'interphase de chacun des lots.

[Thickness of PyC interphases, deposition process and average volume fractions of matrix, fibers and interphase.]

\begin{tabular}{|cccccccc|}
\hline lot & $\begin{array}{c}\text { type de } \\
\text { fibre }\end{array}$ & $\begin{array}{c}\text { mode de } \\
\text { dépôt }\end{array}$ & $\begin{array}{c}\text { épaisseur } \\
\text { interphase } \\
(\mu \mathbf{m})\end{array}$ & $v_{\mathbf{f}}(\%)$ & $v_{\mathbf{m}}(\%)$ & $v_{\mathbf{i}}(\%)$ & $\mathrm{N}$ \\
\hline A & C & P-CVI & 0,5 & 44,8 & 42,3 & 12,9 & 10 \\
\hline B & $"$ & $"$ & 1,0 & 38,6 & 37,2 & 24,2 & 22 \\
\hline C & $"$ & I-CVI & 1,0 & 39 & 37,0 & 24,0 & 17 \\
\hline D & ex-PCS & P-CVI & 0,05 & 64,3 & 35,5 & 0,2 & 9 \\
\hline E & $"$ & $"$ & 0,09 & 65,7 & 32,8 & 1,4 & 15 \\
\hline F & $"$ & $"$ & 0,12 & 56,3 & 41,3 & 2,4 & 18 \\
\hline G & $"$ & $"$ & 0,22 & 47,0 & 49,4 & 3,6 & 18 \\
\hline H & $"$ & $"$ & 1,0 & 57,9 & 32,2 & 9,8 & 9 \\
\hline I & $"$ & I-CVI & 0,1 & 62,4 & 36,5 & 1,0 & 10 \\
\hline
\end{tabular}

* mesures réalisées au MEB à effet de champ $\quad \mathrm{N}$ : nombre de composites unidirectionnels testés

l'aide des équations suivantes :

$$
\begin{gathered}
v_{\mathrm{f}}=100 \frac{V_{\mathrm{f}}}{V_{\text {mèche }}} \equiv 100 \Pi r_{\mathrm{f}}^{2} n L / V_{\text {mèche }} \\
v_{\text {int }}=100 \frac{V_{\text {int }}}{V_{\text {mèche }}} \equiv 100 \Pi n L\left[\left(r_{\mathrm{f}}+e_{\mathrm{i}}\right)^{2}-r_{\mathrm{f}}^{2}\right] / V_{\text {mèche }} \\
V_{\text {mèche }}=V_{\text {mat }}+V_{\text {int }}+V_{\mathrm{f}} \\
v_{\text {mat }}=m_{\text {mat }} / \rho_{\text {mat }}
\end{gathered}
$$




$$
\begin{gathered}
m_{\text {mat }}=m_{\text {mèche }}-m_{\text {int }}-m_{\mathrm{f}} \\
m_{\text {int }}=V_{\text {int }} \rho_{\text {int }} \\
m_{\mathrm{f}}=V_{\mathrm{f}} \rho_{\mathrm{f}}
\end{gathered}
$$

avec $r_{f}$ rayon de la fibre, $n$ nombre de filaments dans la mèche, $L$ longueur de la mèche, $e_{i}$ épaisseur de l'interphase, $V_{\mathrm{i}}$ volume de la phase $\mathrm{i}, m_{\mathrm{i}}$ masse de la phase $\mathrm{i}, \rho_{\mathrm{i}}$ masse volumique de la phase $i,(i=$ mat pour matrice, $i=$ int pour interphase et $i=f$ pour fibre). Le rayon des fibres a été supposé constant et égal à $r_{\mathrm{f}}=3,5 \mu \mathrm{m}$ pour les fibres de carbone et $r_{\mathrm{f}}=7 \mu \mathrm{m}$ pour les fibres ex-PCS. L'incertitude correspondante sur les valeurs des fractions volumiques est inférieure à $5 \%$.

\subsection{ESSAIS MÉCANIQUES}

2.2.1. Procédure d'essai de traction. - Les extrémités des mèches ont été collées dans des tubes en acier selon une technique permettant un centrage précis de l'échantillon (Fig. 4). La longueur de jauge est $20 \mathrm{~mm}$. Les tubes en acier ont été serrés dans les machoires de la machine de traction. Les essais ont été menés avec une vitesse de déplacement constante de 0,1 $\mathrm{mm} / \mathrm{min}$. Un capteur de déplacement monté sur les mors permettait de mesurer l'élongation des mèches.

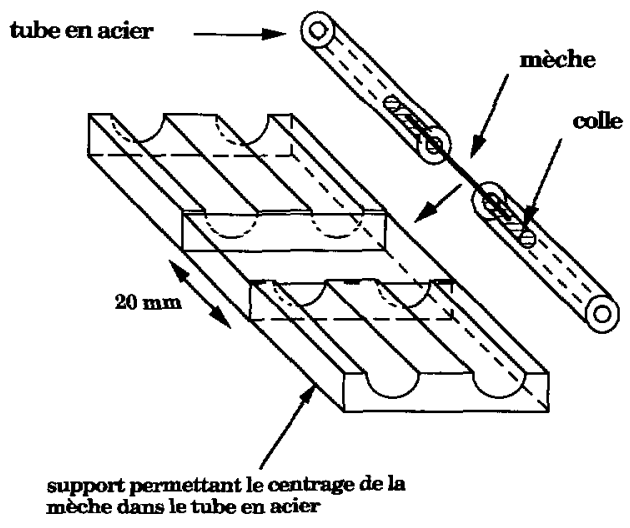

Fig. 4. - Technique de collage de la mèche.

[Preparation of tensile specimens.]

2.2.2. Exploitation des résultats. - Les principales caractéristiques mécaniques du composite telles que les contraintes et déformations, à la limite élastique, à saturation de la fissuration matricielle et à rupture, ont été déduites de la courbe force-déplacement enregistrée lors de chaque essai de traction.

La contrainte à rupture $\left(\sigma_{\mathrm{r}}\right)$ a été calculée en rapportant la force de rupture à la section totale de la mèche, $\left(S_{\text {mèche }}\right)$, en supposant que la matrice contribue à supporter les efforts.

Pour chacun des lots, la dispersion statistique des contraintes à rupture $\sigma_{\mathrm{r}}$ a été décrite à l'aide du module de Weibull $m$ [13]. Les probabilités de rupture associées aux $\sigma_{\mathbf{r}}$ mesurées ont été déterminées grâce à l'estimateur suivant :

$$
P_{\imath}=\frac{i-0,5}{N}
$$




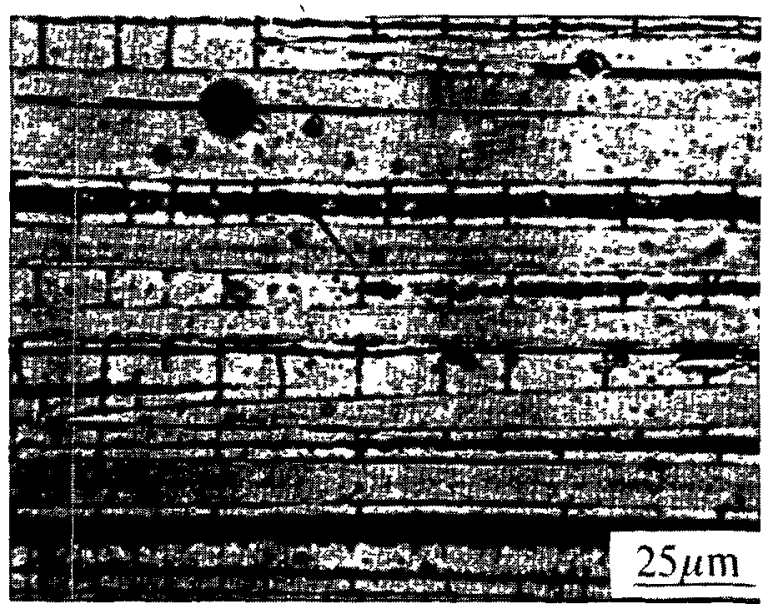

Fig. 5. - Observation au microscope optique du pas de fissuration sur une mèche après rupture en traction (T300/PyC/SiC, lot C).

[Optical micrograph showing matrix crack spacing on a $\mathrm{T} 300 / \mathrm{PyC} / \mathrm{SiC}$ bundle (batch $\mathrm{C}$ ) that failed in tension.]

où $i$ est le rang affecté à chaque mesure de $\sigma_{\mathrm{r}}$ après classement par ordre croissant et $N$ est le nombre total d'échantillons.

Les propriétés de l'interphase ont été caractérisées par :

(i) le pas moyen de fissuration de la matrice à saturation, $d_{s}$, mesuré soit par microscopie optique $\left({ }^{4}\right)$ sur des échantillons enrobés et polis (Fig. 5), soit par microscopie électronique à balayage $\left({ }^{5}\right)$ sur des mèches après rupture en traction (Fig. 6),

(ii) la contrainte de cisaillement interfacial, $\tau_{c}$, qui reflète les transferts de charge entre les fibres et la. matrice, déduite des valeurs de $d_{\mathrm{s}}$ et de la contrainte de saturation, $\sigma_{\mathrm{s}}$, grâce à l'équation suivante proposée par Marshall [14] :

$$
\tau_{\mathrm{c}}=\frac{\sigma_{\mathrm{s}} r_{\mathrm{f}}}{4 v_{\mathrm{f}} d_{\mathrm{s}}\left(1+\frac{E_{\mathrm{f}} v_{\mathrm{f}}}{E_{\mathrm{m}} v_{\mathrm{m}}}\right)}
$$

où $E_{\mathrm{f}}$ et $E_{\mathrm{m}}$ sont les modules d'Young respectifs de la fibre et de la matrice ( $E_{\mathrm{f}_{\text {carbone }}}=200$ $\left.\mathrm{GPa}, E_{\mathrm{f}_{\mathrm{S} 1 \mathrm{C}}}=180 \mathrm{GPa}\right), E_{\mathrm{m}}$ le module d'Young de la matrice $\left(E_{\mathrm{m}}=400 \mathrm{GPa}\right)$. $\sigma_{\mathrm{s}}$ est donnée par la fin du domaine non-linéaire de la courbe force-déplacement.

Cette équation a été préférée à d'autres parce qu'elle n'est pas exprimée en fonction de l'énergie de rupture de la matrice $\Gamma_{m}$ qui est très mal connue pour les matrices $\mathrm{SiC}$ déposées par CVI. Les valeurs de $\tau_{c}$ ainsi obtenues permettent de comparer les différents échantillons entre eux. Elles ne représentent pas la valeur absolue de $\tau$. En effet, il n'existe aucune équation qui prenne en compte la présence d'une interphase souple entre fibres et matrice. Toutes les équations proposées dans la littérature supposent la présence d'une simple interface entre fibre et matrice.

$\left({ }^{4}\right) \mathrm{MeF}_{3}$ de REICHERT-JUNG

$\left({ }^{5}\right)$ JEOL 840 


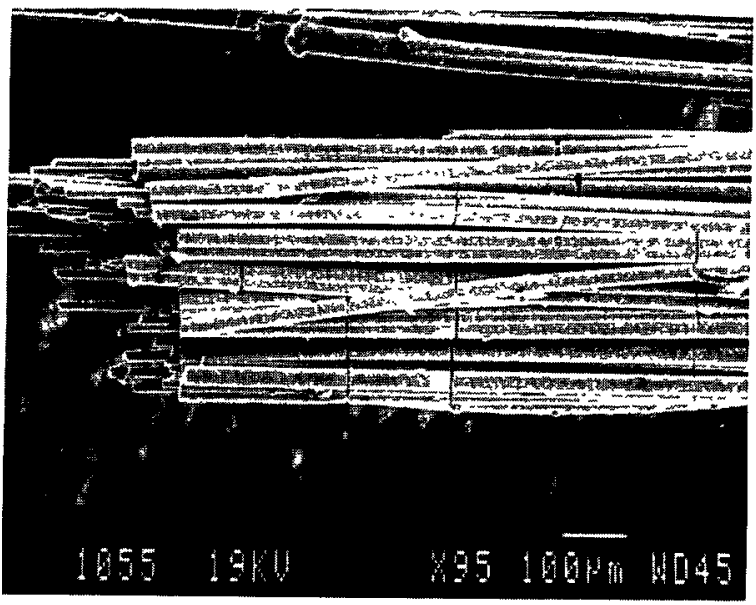

Fig. 6. - Observation au MEB du pas de fissuration sur une mèche après rupture en traction (fibre ex-PCS/PyC/SiC, lot E).

[SEM micrograpb showing matrix crack spacing on a bundle (ex-PCS fiber/PyC/SiC, batch $\mathrm{E}$ ) failed in tension.]

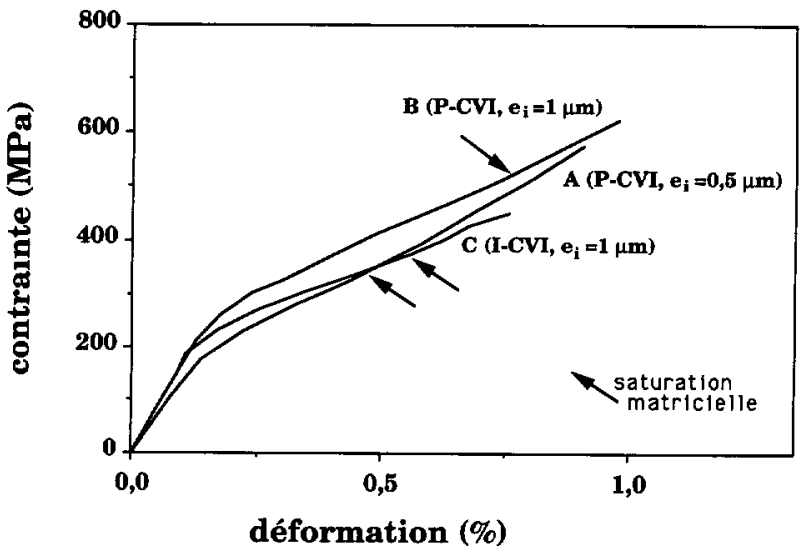

Fig. 7. - Courbes types de comportement en traction des lots $\mathrm{A}, \mathrm{B}, \mathrm{C}$ (fibres carbone/PyC/SiC). [Typical tensile stress-strain curves for specimens from batches A, B, C (T300/PyC/Sic).]

\section{Résultats et discussion}

\subsection{Composites unidirectionnels $\mathrm{C} / \mathrm{PyC} / \mathrm{SiC}$}

3.1.1. Analyse des courbes contrainte-déplacement. - Les courbes types contraintedéplacement caractéristiques de chacun des lots de composites $\mathrm{C} / \mathrm{PyC} / \mathrm{SiC}$ sont présentées sur la figure 7. Les éprouvettes des trois lots présentent le comportement classique des composites à matrice fragile. Trois domaines apparaissent sur les courbes :

(i) un domaine linéaire réduit en raison de la présence de fissures dans la matrice après élaboration et avant essai en raison de la différence des coefficients de dilatation axiaux $\left(\alpha_{a_{\text {matrice }}}-\right.$ $\left.\alpha_{\mathrm{a}_{\mathrm{fibre}}}=4,6 \times 10^{-6}\right)$ et radiaux $\left(-15 \times 10^{-6}<\alpha_{\mathrm{r}_{\text {matrice }}}-\alpha_{\mathrm{r}_{\mathrm{fibre}}}<-20 \times 10^{-6}\right)$ de la matrice 
et de la fibre [15-18], (ii) un domaine non linéaire induit par la fissuration supplémentaire de la matrice et (iii) un domaine linéaire dans la dernière partie de la courbe résultant de la déformation des fibres. A noter que lors de la quasi totalité des essais de traction, le seuil de saturation matricielle a été atteint.

Les composites appartenant au lot C (I-CVI) se distinguent de ceux des lots A et B (P-CVI) par une déformation et une contrainte à rupture plus faibles. L'emploi d'une épaisseur de PyC (P-CVI) plus importante pour le lot $\mathrm{B}$ par rapport au lot $\mathrm{A}$ permet d'accroître légèrement déformation et contrainte à rupture.

3.1.2. Propriétés interfaciales. - Les valeurs moyennes des grandeurs mécaniques calculées à partir des courbes force-déplacement sont reportées sur le tableau II.

Tableau II. - Résultats des lots $A, B, C$ (fibres carbone T300/PyC/SiC).

[Mechanical data for specimens from batches $\mathrm{A}, \mathrm{B}, \mathrm{C},(\mathrm{C} / \mathrm{Pyc} / \mathrm{SiC})$.]

\begin{tabular}{|c|c|c|c|c|c|c|c|c|c|}
\hline lot & $\underset{(\mathrm{MPa})}{\boldsymbol{\sigma}_{\mathbf{r}}}$ & $\mathbf{m}$ & $\underset{(\mathbf{M P a})}{\boldsymbol{\sigma}_{\mathbf{e}}}$ & $\underset{(\mathbf{M P a})}{\boldsymbol{\sigma}_{\mathbf{S}}}$ & $\boldsymbol{\varepsilon}_{\%}$ & $\boldsymbol{E}_{\%}$ & $\underset{\%}{\boldsymbol{E}_{\mathbf{r}}}$ & $\underset{(\mu \mathrm{m})}{\mathrm{ds}}$ & $\tau_{(\mathbf{M P a})}$ \\
\hline$\underset{\substack{\left.\text { (P.CVI, } \\
\mathbf{e}_{\mathrm{i}}=0,5 \mu \mathrm{m}\right)}}{\mid}$ & $\begin{array}{c}570 \\
{[107]}\end{array}$ & 5,9 & $\begin{array}{r}150 \\
{[62]}\end{array}$ & $\begin{array}{r}415 \\
{[78]}\end{array}$ & $\begin{array}{c}0,09 \\
{[0,03]}\end{array}$ & $\begin{array}{c}\mathbf{0 , 5 5} \\
{[\mathbf{0 , 1 1}]}\end{array}$ & $\begin{array}{c}\mathbf{0 , 9 0} \\
{[0,12]}\end{array}$ & 90 & $\begin{array}{l}13,3 \\
{[2,1]}\end{array}$ \\
\hline \begin{tabular}{|c|}
$\mathbf{B}$ \\
$\mathbf{( P . C V I}$, \\
$\left.\mathbf{e}_{\mathbf{i}}=1 \mu \mathrm{m}\right)$
\end{tabular} & $\begin{array}{l}605 \\
{[85]}\end{array}$ & $\mathbf{9 , 3}$ & $\begin{array}{c}164 \\
{[21]}\end{array}$ & $\begin{array}{c}319 \\
{[\mathbf{5 3}]}\end{array}$ & $\begin{array}{c}0,13 \\
{[0,03]}\end{array}$ & $\begin{array}{c}0,46 \\
{[0,12]}\end{array}$ & $\begin{array}{c}1,0 \\
{[0,17]}\end{array}$ & 30 & $\begin{array}{l}32,5 \\
{[6,7]}\end{array}$ \\
\hline $\begin{array}{c}\mathbf{C} \\
\text { (I.CVI, } \\
\left.\mathbf{e}_{\mathbf{i}}=1 \mu \mathrm{m}\right)\end{array}$ & $\begin{array}{l}416 \\
{[85]}\end{array}$ & 6,2 & $\begin{array}{l}132 \\
{[34]}\end{array}$ & $\begin{array}{l}305 \\
{[32]}\end{array}$ & $\begin{array}{l}0,09 \\
{[0,03]}\end{array}$ & $\begin{array}{l}\mathbf{0 , 5 1} \\
{[\mathbf{0 , 1 0}]}\end{array}$ & $\begin{array}{l}0,78 \\
{[0,25]}\end{array}$ & 50 & $\begin{array}{l}20,3 \\
{[2,1]}\end{array}$ \\
\hline
\end{tabular}

[ ] écart type

Les caractéristiques mécaniques moyennes des mèches du lot $\mathrm{B}$ (P- CVI, $e_{\mathrm{i}}=1 \mu \mathrm{m}$ ) sont supérieures à celles des mèches du lot A (P-CVI, $\left.e_{\mathrm{i}}=0,5 \mu \mathrm{m}\right)$, mais de façon peu significative, cependant, le lot $\mathrm{B}$ présente une dispersion plus faible (module de Weibull des contraintes à rupture plus élevé).

La différence notable entre ces deux lots se situe au niveau du pas moyen de fissuration qui est trois fois plus petit dans le cas du lot $B$, ce qui se traduit par une contrainte interfaciale environ 2,7 fois plus élevée que celle du lot $\mathrm{A}$. Cette variation de $d_{\mathrm{s}}$ et $\tau_{\mathrm{c}}$ peut être en partie attribuée à l'influence de $e_{\mathrm{i}}$ sur les contraintes résiduelles après élaboration. Une simulation numérique [19] utilisant un modèle dérivé de celui d'Eshelby [20] a permis le calcul des contraintes résiduelles d'origine thermique dans le cas de microcomposites $\mathrm{C} / \mathrm{PyC} / \mathrm{SiC}$ (Fig. 8) en respectant les épaisseurs moyennes de matrice et d'interphase mesurées sur les lots $\mathrm{A}, \mathrm{B}$ et $\mathrm{C}$. Les valeurs des modules élastiques $\left(E_{1}=12 \mathrm{GPa}, E_{3}=30 \mathrm{GPa}\right.$ et $\left.G=2 \mathrm{GPa}\right)$ [21] de l'interphase PyC (P-CVI) ont été considérées, en première approximation, identiques à celles de l'interphase PyC (I-CVI).

La figure 9 présente les variations des contraintes résiduelles calculées pour deux microcomposites $\mathrm{C} / \mathrm{PyC} / \mathrm{SiC}$ possédant une interphase d'épaisseurs $e_{\mathrm{i}}=0,5 \mu \mathrm{m}$ (Fig. 9a) et $e_{\mathrm{i}}=1 \mu \mathrm{m}$ (Fig. 9b). L'étendue de la zone de décohésion interfaciale est fonction des contraintes résiduelles radiales $\sigma^{\mathrm{R}}$ Les contraintes résiduelles radiales favorisent un frettage de la fibre lorsqu'elles sont négatives ou une décohésion fibre/interphase lorsqu'elles sont positives. La figure 9 montre que, quelle que soit la valeur de $e_{\mathbf{i}}, \sigma^{\mathrm{R}}$ est positive (traction) dans l'interphase et que sa valeur est d'autant plus élevée que $e_{\mathrm{i}}$ est faible : $\sigma^{\mathrm{R}}$ augmente de $20 \%$ lorsque $e_{\mathrm{i}}$ passe de $1 \mu \mathrm{m}$ à 


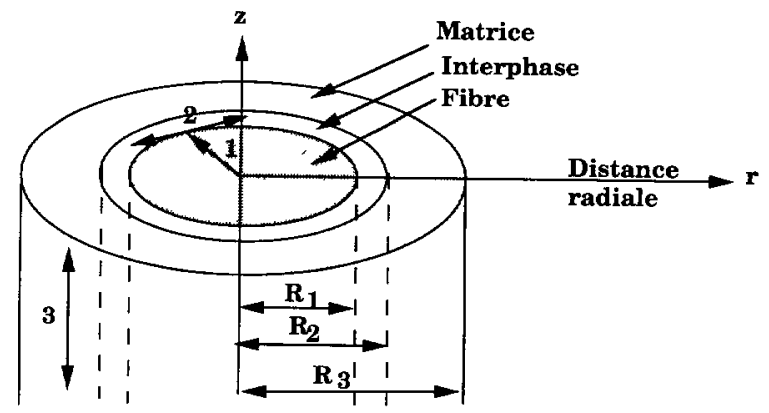

$$
\begin{aligned}
& 1=\text { Contraintes radiales }\left(\sigma^{\mathrm{R}}\right) \\
& 2=\text { Contraintes circonférentielles }\left(\sigma^{\mathrm{H}}\right) \\
& \mathbf{3}=\text { Contraintes longitudinales ou axiales }\left(\sigma^{\mathrm{A}}\right)
\end{aligned}
$$

Fig. 8. - Représentation schématique d'un microcomposite et directions principales des contraintes résiduelles d'origine thermique.

[Schematic diagram showing a microcomposite and defining the directions of thermally induced residual stresses.]

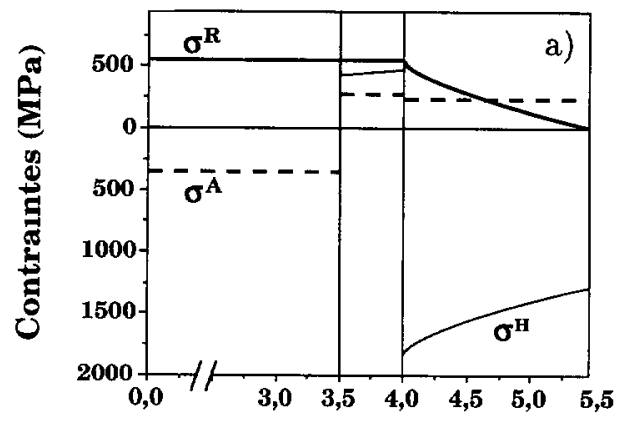

Distance radiale $(\mu \mathrm{m})$

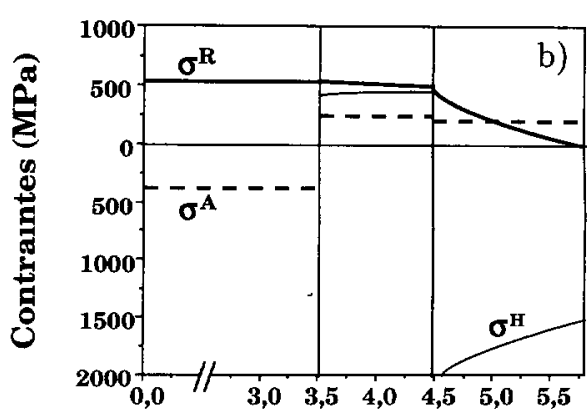

Distance radiale $(\mu \mathrm{m})$

Fig. 9. - Comparaison entre la distribution des contraintes résiduelles d'origine thermique pour des microcomposites C/PyC/SiC. a) $e_{1}=0,5 \mu \mathrm{m}$ et b) $e_{1}=1 \mu \mathrm{m}$.

[Residual stress field in C/PyC/SiC microcomposites. a) $e_{1}=0.5 \mu \mathrm{m}$ and b) $e_{1}=1 \mu \mathrm{m}$.]

$0,5 \mu \mathrm{m}$. La décchésion fibre/interphase doit donc être moindre ( $d_{s}$ plus petit) et les transferts de charge meilleurs ( $\tau_{\mathrm{c}}$ plus élevée) lorsque $e_{\mathrm{i}}=1 \mu \mathrm{m}$. Les résultats du calcul des contraintes résiduelles permettent donc d'expliquer en partie les différences entre les valeurs de $d_{\mathbf{s}}$ mesurées pour le lot $\mathrm{A}$ et le lot $\mathrm{B}$. En effet, la variation du pas de fissuration observée $\left(d_{\mathrm{s}}\right.$ est multiplié par trois lorsque $e_{\mathrm{i}}$ passe de $1 \mu \mathrm{m}$ à $0,5 \mu \mathrm{m}$ ) ne peut être attribuée exclusivement à l'influence modeste de $e_{\mathbf{i}}$ sur $\sigma^{\mathrm{R}}$ lorsqu'elle diminue de $1 \mu \mathrm{m}$ à $0,5 \mu \mathrm{m}$.

Les valeurs moyennes des caractéristiques mécaniques des mèches du lot $\mathrm{C}\left(\mathrm{I}-\mathrm{CVI}, e_{\mathrm{i}}=1 \mu \mathrm{m}\right)$ sont plus faibles et plus dispersées (module de Weibull plus faible) que celles du lot B (P-CVI, $e_{\mathrm{i}}=1 \mu \mathrm{m}$ ). (Pour le lot $\mathrm{C}, \sigma_{\mathrm{r}}=416 \mathrm{MPa}$ alors que pour le lot $\mathrm{B}, \sigma_{\mathrm{r}}=605 \mathrm{MPa}$, soit une différence de $45 \%$ ). Les calculs de contraintes résiduelles effectués sur des microcomposites possédant les mêmes épaisseurs d'interphase et de matrice que les mèches du lot $\mathrm{C}$ indiquent 
que les performances de ce lot devraient être proches de celles des mèches du lot $B$, ce qui est en opposition avec les résultats expérimentaux. Ceci conduit à penser que les valeurs des grandeurs thermomécaniques de l'interphase P-CVI sont différentes de celles de l'interphase I-CVI. En effet, le calcul montre que pour obtenir des contraintes résiduelles compatibles avec les variations de $d_{\mathbf{s}}$, il est nécessaire d'augmenter l'anisotropie des modules d'Young de l'interphase $\mathrm{PyC}$ : le rapport $E_{3} / E_{1} \approx 4,0$ pour l'interphase PyC P-CVI alors que $E_{3} / E_{1} \approx 2,5$ pour celle obtenue en I-CVI. Il est intéressant de noter que cette augmentation de l'anisotropie de l'interphase P-CVI conduit à une plus forte variation des contraintes résiduelles radiales avec $e_{\mathrm{i}}$, qui permettrait d'expliquer la forte variation de $d_{\mathrm{s}}$ obtenue lorsque $e_{\mathrm{i}}$ P-CVI diminue (lots $A$ et $B$ ).

Cette différence des grandeurs thermomécaniques peut s'expliquer par la structure particulière du $P y C$ obtenu en $P-C V I$. En effet, une étude précédente [11] a montré que les dépôts P-CVI réalisés dans les mêmes conditions expérimentales présentent les caractéristiques suivantes :

(i) longueur des couches de carbone très importante (jusquà $15 \mathrm{~nm}$ ), (ii) faible empilement des couches et (iii) couches orientées parallèlement à l'axe de la fibre. La croissance des couches de carbone est dite "latérale" (Fig. 10a).

Les dépôts I-CVI possèdent une structure tout à fait opposée car la longueur des couches est plus réduite $(<5 \mathrm{~nm})$ et celles-ci ont tendance à s'empiler selon une direction perpendiculaire à l'axe de la fibre, la croissance est dite "colonnaire" (Fig. 10b).

On peut penser que la structure orientée du pyrocarbone P-CVI confère à l'interphase une rigidité accrue et une énergie de rupture plus faible par rapport à l'interphase I-CVI. La rigidité accrue favorise les transferts d'effort sur la matrice. La distance entre les fissures matricielles $d_{\mathrm{s}}$ doit donc être plus faible ainsi que cela a été observé. L'énergie de rupture plus faible favorise la décohésion. Cependant, si la longueur de décohésion interfaciale était plus grande, $d_{\mathrm{s}}$ serait plus importante, ce qui serait en désaccord avec la conclusion précédente et les observations. Des images TEM montrant la position des fissures au sein de l'interphase permettraient de conclure de manière indiscutable sur cette question. Ainsi que cela a été observé dans certains interphases de pyrocarbone [22], on peut penser qu'une énergie de décohésion plus faible permet la formation de plusieurs fissures parallèles au sein de l'interphase, lesquelles sont plus courtes que lorsqu'une seule fissure se forme à l'interface entre la fibre et l'interphase. La présence d'un tel système de fissures dans les interphases P-CVI serait alors compatible avec le faible pas de fissuration observé à saturation.

\subsection{Composites unidirectionnels $\mathrm{SiC} / \mathrm{PyC} / \mathrm{SiC}$}

3.2.1. Analyse des courbes contrainte-déplacement. - Les courbes types contraintedéplacement, caractéristiques des lots D à I, sont présentées sur la figure 11. Les trois domaines observés habituellement sont présents. Le domaine élastique est beaucoup plus étendu que dans le cas des mèches $\mathrm{C} / \mathrm{PyC} / \mathrm{SiC}$. Le domaine de multifissuration est très large, sauf pour le lot $\mathrm{D}$ qui ne semble pas atteindre la saturation matricielle. Les mèches des lots $\mathrm{E}, \mathrm{F}$ présentent un comportement similaire. Deux lots se mettent en évidence : le lot $\mathrm{D}$ qui subit une rupture prématurée sous une faible contrainte et le lot $G$ dont l'allongement et la contrainte à rupture sont beaucoup plus élevés que pour les autres lots.

3.2.2. Propriétés interfaciales. - Les valeurs des grandeurs mécaniques calculées à partir des courbes contrainte-déplacement sont rassemblées sur le tableau III.

Il apparaît que le lot $\mathrm{D}$ présente les résultats les plus dispersés, alors que les résultats des lots $\mathrm{E}$ et $\mathrm{G}$ sont très groupés.

L'influence propre de l'épaisseur de l'interphase sur les propriétés mécaniques est difficile à déterminer car les fractions volumiques de matrice et de fibres varient d'un lot à l'autre. Cepen- 

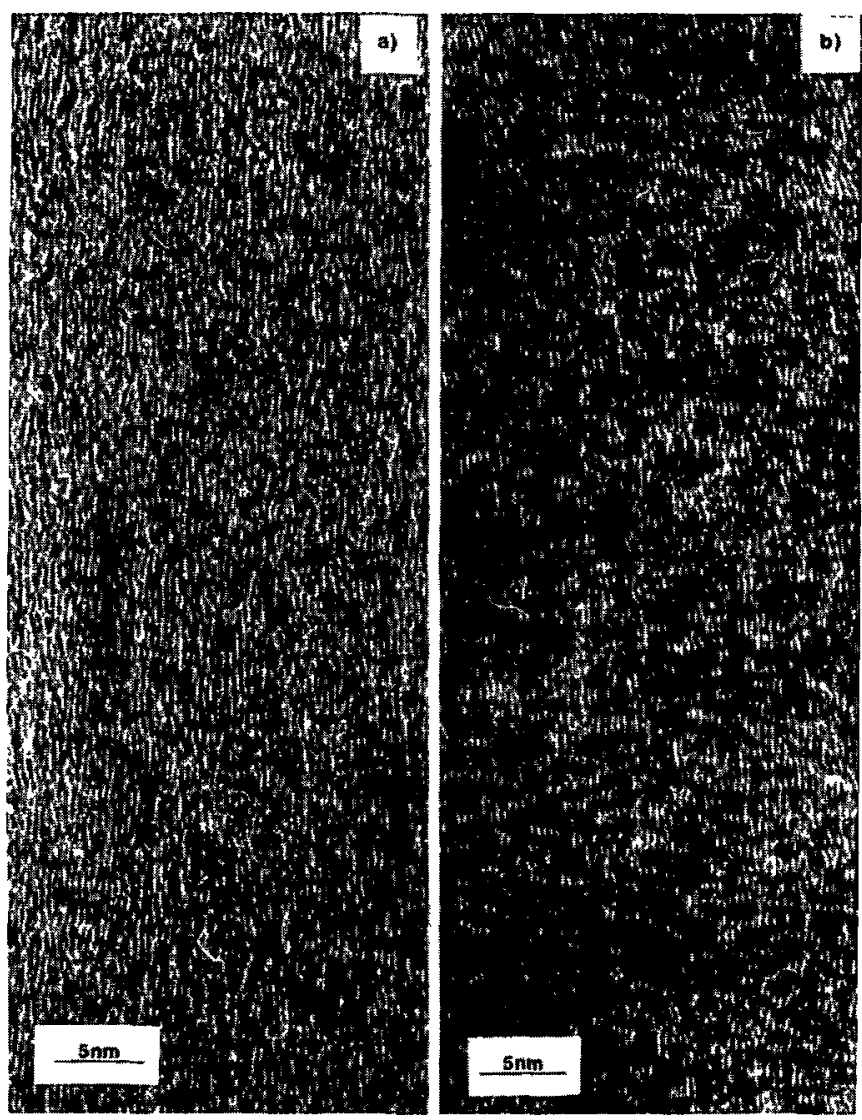

Fig. 10. - Comparaison de la structure des PyC obtenus en I-CVI et P-CVI : a) P-CVI, $T=1000^{\circ} \mathrm{C}$, $P=3 \mathrm{kPa}$ et $t_{\mathrm{R}}=10 \mathrm{~s}$ (montrant une croissance latérale) et b) I-CVI, $T=1050^{\circ} \mathrm{C}, P=4,7 \mathrm{kPa}$ et $Q=40 \mathrm{sccm}$ (montrant une croissance colonnaire).

[HRTEM micrographs of the PyC interphases deposited by I-CVI and P-CVI: a) P-CVI, $T=1000^{\circ} \mathrm{C}$, $P=3 \mathrm{kPa}$ and $t_{\mathrm{R}}=10 \mathrm{~s}$ (lateral growth) and b) I-CVI, $T=1050^{\circ} \mathrm{C}, P=4,7 \mathrm{kPa}$ and $Q=40 \mathrm{sccm}$ (columnar growth).]

dant, l'ensemble des caractéristiques mécaniques des composites unidirectionnels SiC/PyC (P$\mathrm{CVI} / \mathrm{SiC}$ dépendent de la valeur de $e_{\mathrm{i}}$, passant par un optimum pour le lot $\mathrm{G}\left(e_{\mathrm{i}}=0,22 \mu \mathrm{m}\right)$. Ceci est en bon accord avec divers travaux montrant une évolution semblable des contraintes à rupture avec l'épaisseur d'interphase PyC obtenue par I-CVI dans le cas de matrices SiC ou vitrocéramique $[5,6,23]$.

Les contraintes thermiques résiduelles ont été calculées dans le cas de microcomposites $\mathrm{SiC} / \mathrm{PyC} / \mathrm{SiC}$ pour différentes épaisseurs d'interphase $\left(0,05 \mu \mathrm{m}<e_{\mathrm{i}}<1 \mu \mathrm{m}\right)$, une épaisseur de matrice fixée à $4 \mu \mathrm{m}$ (Fig. 12) et un rapport $E_{1} / E_{3}=2,5$ pour l'interphase pyrocarbone. Cette simulation numérique montre que la contrainte longitudinale dans la matrice (qui influence la limite d'élasticité $\epsilon_{\mathrm{e}}$ ) diminue légèrement lorsque $e_{\mathrm{i}}$ croît. $\epsilon_{\mathrm{e}}$ devrait donc augmenter avec $e_{\mathrm{i}}$, ce qui est effectivement observé pour les lots $D, E$ et $F$. Dans le cas du lot $H\left(e_{i}=1 \mu \mathrm{m}\right) \epsilon_{\mathrm{e}}$ est anormalement faible. La raison exacte n'a pas pu être élucidée. 


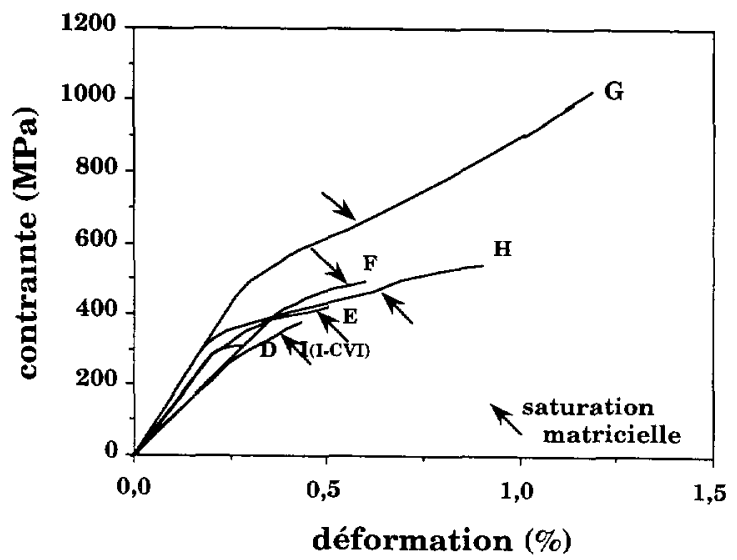

Fig. 11. - Courbes types de comportements en traction des lots D, E, F, G, H, I (fibres ex$\mathrm{PCS} / \mathrm{PyC} / \mathrm{SiC}$ ).

[Typical tensile stress-strain curves for specimens from batches D, E, F, G, H, I (ex-PCS fibres/PyC/SiC).]

Tableau III. - Résultats des lots D, E, F, G, H, I et de la référence [18] (fibres ex$\mathrm{PCS} / \mathrm{PyC} / \mathrm{SiC}$ ).

[Mechanical data for specimens from batches D, E, F, G, H, I and from Reference [18] (ex-PCS fibers/PyC/SiC).]

\begin{tabular}{|c|c|c|c|c|c|c|c|c|c|}
\hline lot & $\underset{(\mathbf{M P a})}{\boldsymbol{\sigma}_{\mathbf{r} \mathbf{1}}}$ & $\mathbf{m}$ & $\underset{(\mathrm{MPa})}{\boldsymbol{\sigma}_{\mathbf{e}}}$ & $\underset{\mathbf{M P a})}{\sigma_{\mathbf{M P a}}}$ & $\underset{\%}{\boldsymbol{E}_{\mathbf{e}}}$ & $\underset{\%}{\boldsymbol{E}_{\mathbf{s}}}$ & $\underset{\sigma_{0}}{\boldsymbol{E}}$ & $\underset{(\mu \mathrm{m})}{\mathbf{d s}}$ & $\underset{(\mathbf{M P a})}{\tau_{\mathbf{c}}}$ \\
\hline $\begin{array}{c}\mathbf{D} \\
\begin{array}{c}\text { (P.CVI, } \\
\left.\mathbf{e}_{\mathrm{i}}=\mathbf{0}, \mathbf{0 5} \mu \mathrm{m}\right)\end{array}\end{array}$ & $\begin{array}{c}300 \\
{[1.20]}\end{array}$ & 2,6 & $\begin{array}{c}228 \\
{[127]}\end{array}$ & --- & $\begin{array}{c}0,18 \\
{[0,07]}\end{array}$ & -- & $\begin{array}{c}0,26 \\
{[0,04]}\end{array}$ & 200 & --- \\
\hline $\begin{array}{c}\mathbf{E} \\
(\mathbf{P}-\mathrm{CV}, \\
\left.\mathbf{e}_{\mathrm{i}}=0,09 \mu \mathrm{m}\right)\end{array}$ & $\begin{array}{c}470 \\
{[106]}\end{array}$ & 10,9 & $\begin{array}{r}\mathbf{3 0 0} \\
{[\mathbf{3 8}]}\end{array}$ & $\begin{array}{l}\mathbf{3 7 0} \\
{[58]}\end{array}$ & $\begin{array}{c}0,21 \\
{[0,04]}\end{array}$ & $\begin{array}{r}0,35 \\
{[0,11}\end{array}$ & $\mid \begin{array}{c}0,65 \\
{[0,20]}\end{array}$ & 180 & 18,0 \\
\hline $\begin{array}{c}F \\
\text { (P.CVI, } \\
\left.e_{\mathrm{i}}=0,12 \mu \mathrm{m}\right)\end{array}$ & $\begin{array}{c}549 \\
{[58]}\end{array}$ & 5,5 & $\begin{array}{l}386 \\
{[52]}\end{array}$ & $\begin{array}{l}472 \\
{[31]}\end{array}$ & $\begin{array}{c}0,31 \\
{[0,10]}\end{array}$ & $\begin{array}{c}0,40 \\
{[0,08]}\end{array}$ & $\begin{array}{c}\mathbf{0 , 6 4} \\
{[\mathbf{0 , 1 6}}\end{array}$ & 70 & 43,0 \\
\hline $\begin{array}{c}\mathbf{G} \\
(\mathrm{P}-\mathrm{CVI}, \\
\left.\mathrm{e}_{\mathrm{i}}=\mathbf{0}, 22 \mu \mathrm{m}\right)\end{array}$ & $\begin{array}{c}939 \\
{[144]}\end{array}$ & 7,9 & $\begin{array}{l}387 \\
{[29]}\end{array}$ & $\begin{array}{l}566 \\
{[67]}\end{array}$ & $\mid \begin{array}{c}0,26 \\
{[0,05]}\end{array}$ & $\begin{array}{c}0,58 \\
{[0,10]}\end{array}$ & $\left|\begin{array}{c}1,30 \\
{[0,16}\end{array}\right|$ & 30 & 90,7 \\
\hline $\begin{array}{c}\mathbf{H} \\
(\mathrm{P}-\mathrm{CVI}, \\
\mathbf{e}_{\mathbf{i}=1,0 \mu \mathrm{m})}\end{array}$ & $\begin{array}{l}470 \\
{[87]}\end{array}$ & 6,0 & $\begin{array}{l}200 \\
{[44]}\end{array}$ & $\begin{array}{c}304 \\
{[47]}\end{array}$ & $\begin{array}{c}0,17 \\
{[0,03]}\end{array}$ & $\begin{array}{c}0,43 \\
{[0,12]}\end{array}$ & $\begin{array}{c}0,97 \\
{[0,15]}\end{array}$ & 260 & 10,2 \\
\hline $\begin{array}{c}\text { I } \\
(\mathrm{I}-\mathrm{CVI}, \\
\left.\mathrm{e}_{\mathrm{i}}=0,1 \mathrm{~m}\right)\end{array}$ & $\begin{array}{l}408 \\
{[76]}\end{array}$ & 6,2 & $\begin{array}{l}280 \\
{[57]}\end{array}$ & $\begin{array}{c}\mathbf{3 4 7} \\
{[53]}\end{array}$ & $\begin{array}{c}0,21 \\
{[0,05]}\end{array}$ & $\begin{array}{c}0,31 \\
{[0,03]}\end{array}$ & $\left|\begin{array}{c}0,46 \\
{[0,12]}\end{array}\right|$ & 70 & 37,5 \\
\hline $\begin{array}{c}{[23]} \\
(\mathrm{I}-\mathrm{CVI}, \\
\left.\mathrm{e}_{\mathrm{i}}=0,2 \mu \mathrm{m}\right)\end{array}$ & $\begin{array}{l}458 \\
{[82]}\end{array}$ & $\cdots$ & $\begin{array}{l}237 \\
{[42]}\end{array}$ & $\cdots$ & 0,081 & --- & $0,63 \mid$ & 35 & --- \\
\hline
\end{tabular}

[ ] écart type 

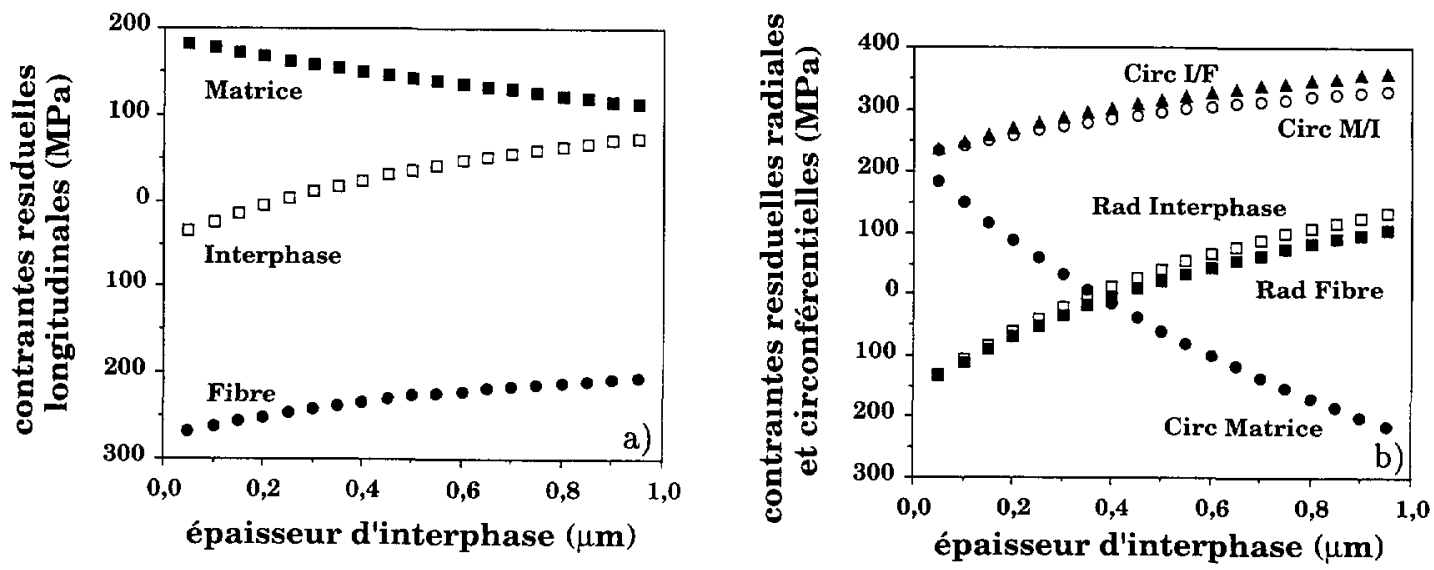

Fig. 12. - Variations des contraintes résiduelles d'origine thermique dans un microcomposite $\mathrm{SiC} / \mathrm{PyC} / \mathrm{SiC}$ en fonction de l'épaisseur de l'interphase pyrocarbone : a) contraintes longitudinales et b) contraintes radiales et circonférentielles.

[Variations of thermally induced residual stresses in a $\mathrm{SiC} / \mathrm{PyC} / \mathrm{SiC}$ microcomposite as a function of the thickness of $\mathrm{PyC}$ interphases: a) axial stresses and b) radial and circumferantial stresses.]

Les contraintes résiduelles radiales dans l'interphase sont de compression pour $e_{\mathrm{i}}<0,4 \mu \mathrm{m}$ et de tension pour $e_{\mathrm{i}}>0,4 \mu \mathrm{m}$. Les contraintes résiduelles de compression assurant le frettage de la fibre et de l'interphase par la matrice, limitent la décohésion interfaciale fibre/interphase et favorisent le transfert de charge fibre/matrice. Inversement, les contraintes résiduelles de tension, favorisent les décohésions ce qui explique l'évolution de $d_{\mathrm{s}}$ et de $\tau_{\mathrm{c}}$ avec $e_{\mathrm{i}}$ à déformation à rupture comparable.

La comparaison des résultats obtenus à épaisseur d'interphase constante pour les mèches $\mathrm{du}$ lot I (I-CVI, $\left.e_{\mathrm{i}}=0,1 \mu \mathrm{m}\right)$ et des lots $\mathrm{E}$ et F (P-CVI, $e_{\mathrm{i}}=0,09$ et $\left.0,12 \mu \mathrm{m}\right)$ montre qu'une interphase P.CVI améliore les propriétés mécaniques. Cette amélioration est confortée par la comparaison des résultats obtenus pour le lot $\mathrm{G}\left(\mathrm{P}-\mathrm{CVI}, e_{\mathrm{i}}=0,22 \mu \mathrm{m}, V_{\mathrm{f}}=47 \%\right)$ avec ceux obtenus par Fretty [24] pour des composites $\mathrm{SiC} / \mathrm{PyC} / \mathrm{SiC}$ unidirectionnels (I-CVI, $e_{\mathrm{i}}=0,2 \mu \mathrm{m}, V_{\mathrm{f}}=45 \%$, longueur $=50 \mathrm{~mm} ;$ Tab. III). Les valeurs moyennes de contrainte et d'allongement à rupture sont nettement supérieures pour le lot G. La différence de longueur de jauge ne permet pas d'expliquer l'écart entre les résultats. D'ailleurs Fretty obtient des mesures peu différentes avec des composites unidirectionnels d'une longueur de $180 \mathrm{~mm}$. Il est donc probable que l'interphase P-CVI est à l'origine de l'amélioration des propriétés mécaniques. Ceci peut être attribué à la microstructure particulière du PyC déposé en P-CVI. Comme dans le cas des composites $\mathrm{C} / \mathrm{PyC} / \mathrm{SiC}$, les calculs de contraintes résiduelles semblent en accord avec les valeurs expérimentales de $d_{\mathrm{s}}$ lorsque l'on considère un degré d'anisotropie supérieur pour l'interphase P-CVI $\left(E_{1} / E_{3} \approx 5\right)$ par rapport à l'interphase I-CVI $\left(E_{1} / E_{3} \approx 2,5\right)$. Si l'on admet que le degré d'anisotropie va de pair avec l'orientation des couches, les observations précédentes relatives aux composites $\mathrm{C} / \mathrm{PyC} / \mathrm{SiC}$ s'appliquent.

\section{Conclusion}

Les deux familles de mèches $(\mathrm{C} / \mathrm{PyC} / \mathrm{SiC}$ et $\mathrm{SiC} / \mathrm{PyC} / \mathrm{SiC})$ présentent le comportement classique de composites à matrice céramique sollicités en traction.

La comparaison des propriétés mécaniques (contraintes et déformations de fissuration, de saturation, et de rupture) des différents lots de mèches a permis de mettre en évidence une forte 
variation des propriétés mécaniques avec l'épaisseur de l'interphase PyC (P-CVI), surtout dans le cas de mèches $\mathrm{SiC} / \mathrm{PyC} / \mathrm{SiC}$. L'épaisseur de l'interphase semble avoir une influence moindre dans le cas de mèches $\mathrm{C} / \mathrm{PyC} / \mathrm{SiC}$, bien que les valeurs obtenues avec $e_{\mathrm{i}}=1 \mu \mathrm{m}$ soient beaucoup moins dispersées que celles obtenues avec $e_{\mathrm{i}}=0,5 \mu \mathrm{m}$. Des études sur les contraintes résiduelles de microcomposites $\mathrm{C} / \mathrm{PyC} / \mathrm{SiC}$ et $\mathrm{SiC} / \mathrm{PyC} / \mathrm{SiC}$ permettent d'expliquer en partie l'évolution des caractéristiques mécaniques avec $e_{\mathrm{i}}$.

La comparaison des lots à interphase $\mathrm{PyC}$ (P-CVI) et PyC (I-CVI) a montré une nette amélioration des caractéristiques mécaniques lors de l'utilisation d'une interphase $\mathrm{PyC}(\mathrm{P}$ CVI). Ceci semble être lié à la microstructure particulière du $P y C$ déposé en $P-C V I$ [10] qui permettrait un meilleur transfert de charge (augmentation de l'anisotropie) et favoriserait une diminution de l'énergie de rupture au sein de l'interphase elle-même.

\section{Remerciements}

La présente étude a été réalisée avec l'aide de SEP, sous la forme d'une bourse d'étude attribuée à $P$. Dupel et la fourniture de matériaux. Elle a, par ailleurs, bénéficié de l'assistance technique de B. Humez.

\section{Bibliographie}

[1] Naslain R., Introduction aux matériaux composites 2- Matrices métalliques et céramiques, CNRS/IMC Ed., Bordeaux, (1985).

[2] Evans A.G., Zok F.W. et Davis J., The role of interfaces in fiber- reinforced brittle matrix composites, Compos. Scr. Technol. 42 (1991) 3.

[3] Grateau L., Etude du comportement mécanique de composites monofilamentaires SiC/SiC- Rôle de l'interface, Thèse de doctorat, numéro d'ordre : 779 (université Paris -Sud, Centre d'Orsay, décembre 1987).

[4] Naslain R., Dugne O., Guette A., Sevely J., Robin-Brosse C., Rocher J.P. et Cotteret J., Boron nitride in ceramic-matrix composites, J. Am. Ceram. Soc. 74 (1991) 2482.

[5] Menessier E., Guette A., Pailler A. et Naslain R., "Influence de l'épaisseur d'un dépôt pyrocarbone sur les propriétés mécaniques d'un matériaux composite (fibre Nicalon/matrice vitrocéramique)", Comptes rendus des Sixièmes Journées Nationales sur les Composites, Paris, 11-13 octobre 1988, p. 195.

[6] Lowden R.A. et Stinton D.P., The influence of the fiber-matrix bond on the mechanical behaviour of Nicalon/SiC composites, Oak Ridge National Laboratory, Rapport $\mathrm{n}^{\circ}$ ORNL/TM-10667, DE88006155 (1987) p. 44.

[7] Naslain R., "Interfaces et interphases in MMC and CMC : A comparative approach", Actes de Mechanical Properties and Applications of MMC (Japan-France Workshop on MMC Paris, 1992).

[8] Cojean D., Composites $\mathrm{SiC} / \mathrm{C} / \mathrm{SiC}$ : Relation entre les propriétés mécaniques et la microtexture des interphases, Thèse de doctorat, numéro d'odre : 104 (Université de Pau, septembre 1991).

[9] Dupel P., Pailler R. et Naslain R., Pulse chemical vapor deposition (P-CVD) and infiltration (PCVI) of pyrocarbon in model pores with rectangular cross section, Part 2 : Study of infiltration, J. Mat. Sciences 29 (1994) 1056.

[10] Dupel P., Bourrat X. et Pailler R., Anisotropic pyrocarbon obtained at low temperature by pulseCVI : Structural characterization, soumis à Carbon.

[11] Dupel P., Pailler R., Langlais F., Naslain R. et Costecalde A., CVD/CVI of pyrocarbon from propane on flat substrate and in model pores with rectangular cross-sections, soumis à Carbon. 
[12] Heraud L., Christin F. et Naslain R., Brevet Français du 06/09/1977, n FR-A-2 401888.

[13] W.A. Weibull, A statistical distribution of wide applicability, J. Appl. Mech. sept. (1951) 525.

[14] Marshall D.B. et Evans A.G., Failure mechanisms in ceramic- fiber/ceramic-matrix composites, J. Am. Ceram. Soc. 68 (1985) 225.

[15] Menessier E., Dumont J.P., Guette A., Pailler R., Rabardel L. et Naslain R., axial and radial coefficients of thermal expansion of carbon fibers in the $20-430^{\circ} \mathrm{C}$ temperature range as derived from the thermal expansion of 1 D-C-SiO ${ }_{2}\left(\mathrm{~B}_{2} \mathrm{O}_{3}\right)$, Compos. Ceram. Eng. Sci. Proc. 10 (1989) 1439.

[16] Yasuda E., Tanabe T., Machino H. et Takaku A., Thermal expansion behaviour various types of carbon fibers up to $1000^{\circ} \mathrm{C}$, Extended Abstract, 18th Biennal Conf. Carbon, Worester (MA) (Amer. Carbon Soc., ed., University Park PA) p. 30.

[17] Villeneuve J.F., Naslain R., Fourmeaux R. et Sevely J., Longitudinal/radial thermal expansion and poisson ration of some ceramic fibers as measured by TEM, Composite Sci. Technology 49 (1993) 89.

[18] Lowden R.A., Characterization and control of the fiber-matrix interface in ceramic matrix composites, ORNL/TM-11039 (Available NTIS, US Dept. Commerce, Springfield VA, march 1989) p. 26-32.

[19] Bobet J.L. et Lamon J., Thermal residual stress field in continuous fiber reinforced ceramix matrix microcomposites, Proc of MAT-TEC 92, (A. Niku-Lari, ed., Grenoble, 1992) p. 35.

[20] Eshelby J.D., The elastic field outside an ellipsoidal inclusion, Proc of the Royal Society (London, Serie A, 252, 1959) p. 561.

[21] Bobet J.L., Lamon L., Thermal residual stress in Ceramic matrix Composites. Part 1 - Axisymmetrical Model and Finite Element Analysis. Acta Met. sous presse.

[22] Droillard C., Lamon J. et Bourrat X., "Influence of Interfacial Failure Mode on the Stress-Strain Behavior and Fracture Toughness of 2D SiC/SiC Composites with Multilayered Interphases" 1994 Fall Meeting, Materials Research Society (Boston, December 1994).

[23] Pluvinage $P$., Etude expérimentale et simulation numérique du comportement mécanique de matériaux composites $\mathrm{SiC} / \mathrm{SiC}$. Influence des paramètres de stratification et d'élaboration, Thèse de doctorat, numéro d'ordre 584 (Université de Bordeaux I, Avril 1991).

[24] Fretty N., Comportement et endommagement mécaniques de torons densifiés et de matériaux 1D et $2 \mathrm{D} \mathrm{SiC} / \mathrm{SiC}$. Influence de vieillissement sous air à haute température, Thèse de doctorat (Ecole Nationale Supérieure des Mines de Paris, septembre, 1991). 